\title{
Trends and Challenges for Quality of Service and Quality of Experience for Wireless Mesh Networks
}

\author{
Elisangela S. Aguiar ${ }^{1}$, Billy A. Pinheiro', \\ João Fabrício S. Figueirêdo ${ }^{1}$, Eduardo Cerqueira ${ }^{1}$, \\ Antônio Jorge. G. Abelém ${ }^{1}$ and Rafael Lopes Gomes ${ }^{2}$ \\ ${ }^{1}$ Federal University of Pará \\ ${ }^{2}$ State University of Campinas \\ Brazil
}

\section{Introduction}

Wireless communications have received a lot of attention from both industry and academic groups. Wireless access allows independency between the user's position and the physical bearer used to access services from the network, as well as, it supports the delivery of multimedia content ubiquitously (Chiti et al., 2008) (Akyildiz \& Wang, 2009). Nowadays, the Wireless Mesh Network (WMN) model is one of the most relevant approaches to provide lastmile access in emerging communication systems (Held, 2005) (Zhang et al., 2006) (Hossain \& Leung, 2009), such as The Institute of Electrical and Electronic Engineers (IEEE) 802.11s (802.11s, 2010). Another standard that enables mesh mode is the IEEE $802.16(802.16,2010)$, which is used in Worldwide Interoperability for Microwave Access (WiMAX) systems.

WMNs are a special case of ad hoc networks, which allow multiple hops, increase the coverage area, and have low implementation cost and support ubiquitous features for Internet access. A WMN consists of clients (Mesh Clients (MC)), routers (Mesh Routers $(\mathrm{MR})$ ) and gateways, where routers provide connectivity to a set of fixed and/or mobile users and gateways assure Internet connectivity as presented in Figure 1.

Multimedia applications, such as video streaming, Voice over IP (VoIP), and Internet Protocol Television (IPTV), will be abundant in future wireless mesh systems and, consequently, the end-to-end quality level support for these services is a major requirement for a near future. In this context, new Quality of Service (QoS) (Bok-Nyong Park et al. 2006) and Quality of Experience (QoE) (Jain, 2004) approaches are needed to optimize the usage of (scarce wireless) network resources and increase the user's satisfaction.

QoS-based schemes define a set of network level (and packet level) measurement and control operations to guarantee the distribution of multimedia content, in wired and wireless networks, with an acceptable quality level. Traditional QoS metrics, such as packet loss rate, packet delay rate and throughput, are typically used to indicate the impact on the multimedia quality level from the network's point of view, but do not reflect the user's experience. The receiving (by user devices), presenting (by displaying units) and perceiving (by end-users) of the applications are not considered. 
In order overcome the limitations of current QoS schemes, QoE approaches have been introduced. Trends in QoE-based solutions are creating a new paradigm regarding humanbased quality level support in WMNs. QoE measurement operations can be used as an indicator of how a WMN environment meets the end-user needs. Researches on assessment schemes, control mechanisms and wireless resource management approaches based on QoE are being developed and will used as an extension to current QoS solutions (Yamada et al., 2007) (Monteiro \& Nunes, 2007) (De Vleeschauwer et al., 2008).

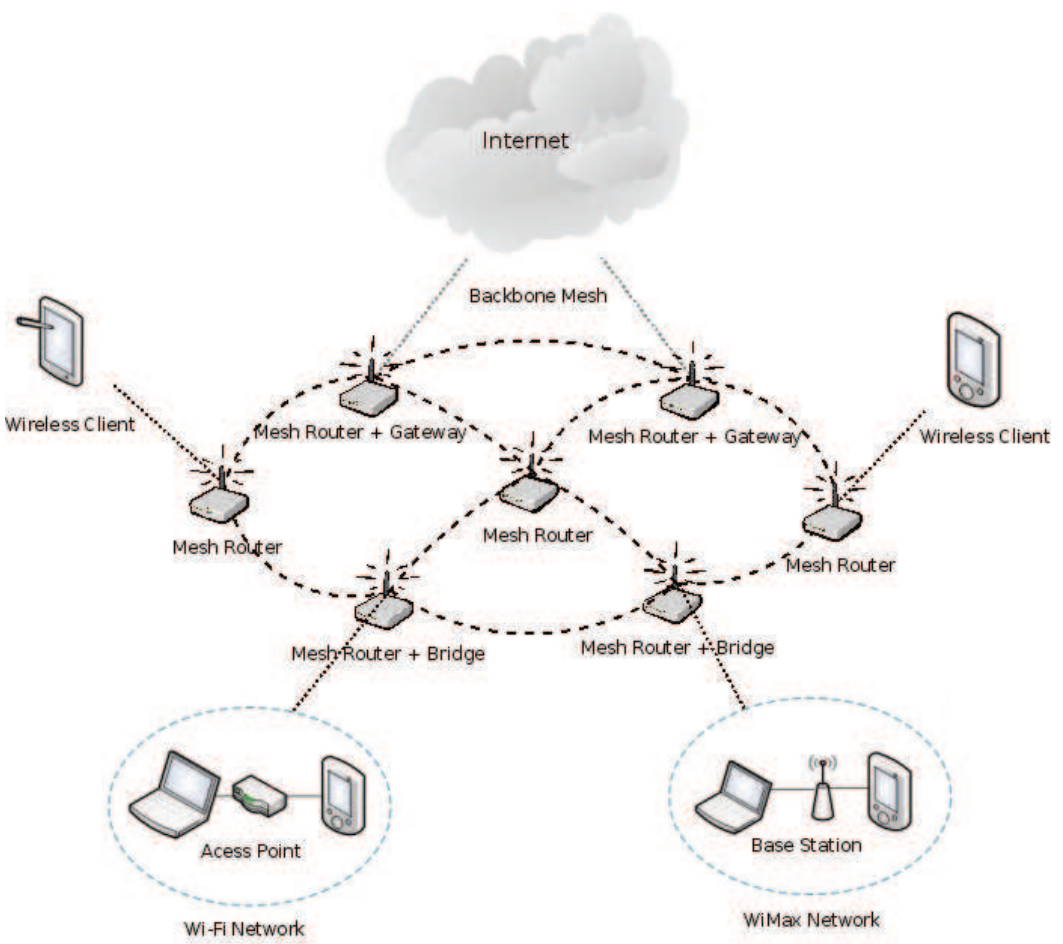

Fig. 1. A Generic Wireless Mesh Network

In this context, the development of an efficient and wise QoS/QoE-based routing scheme is one important challenge for the success of emerging multimedia-aware WMNs. Additionally, novel routing solutions must support a cross-layer approach to improve the overall system performance. Other issues that aim to provide quality level support in WMNs include new admission control, load-balance, resource reservation, overprovisioning, and cognitive radios mechanisms.

In this chapter, an overview of the most relevant challenges and trends in WMNs (focused on IEEE 802.11s) in terms of routing, cross-layer, QoS, and QoE support will be addressed. Due to the importance of routing schemes on optimization operations, a particular attention will be given in this area. To assist on explaining of such challenges, different approaches on QoE and QoS issues will be discussed. In order to show the benefits of cross-layer routing solutions on WMNs, simulation experiments were carried out to present the impact of a new routing scheme on the network's and user's point of view. 
The remainder of this chapter is organized as follows. Section 2 discusses quality of level issues in WMNs. Cross-layer schemes are described in Section 3. The implementation and validation of a WMNs routing solutions are presented in Section 4. Finally, Section 5 presents the final considerations.

\section{Quality of level support in wireless mesh networks}

This section discusses the main approaches to assure quality level support for multimedia applications in wireless mesh networks with focus on QoS and QoE issues.

\subsection{Quality of service issues}

End-to-end quality of service control for fixed and mobile users is a core requirement for the success of emerging wireless systems. This control aims to increase the user satisfaction, while enlarging the revenue to network operators. With this goal in mind, the Internet has been a heavily researched topic in QoS networking for more than one decade. Several QoS models have been proposed with the goal of enriching the Internet with QoS guarantees that the current best effort model cannot support. Each approach defines its own mechanisms and parameters for traffic control and resource management, although usually at different granularities. It is common for a QoS model to be based on the notion of a class as supported by well-know QoS models, such as Differentiated Service (DiffServ), IEEE 802.11e, IEEE 802.16d and Universal Mobile Telecommunication System (UMTS).

Trends in last-mile Internet access require new QoS control mechanisms for IEEE 802.11s (mesh networks). However, the end-to-end QoS support in such scenarios is not trivial and is a research challenge. First of all, they must assure the high capacity needs of the access nodes that have to forward the accumulated traffic of their underling users. Moreover, WMNs have to cope with multiple strict QoS requirements of a large number of multimedia applications, including packet delay, throughput, and packet-error-rate. Finally, they must provide a large enough effective communication range to ensure that no Access Point (AP)s (or groups of APs) are isolated from the Internet gateways. In order to satisfy the above requirements, a set of novel QoS techniques needs to be exploited, such technology enablers include but not limited to multi-hopping, various multiple antennas techniques, novel Medium Access Control (MAC), resource reservation, over provisioning, admission control schemes and routing, where the last one will be explored in this chapter.

Another issue to be investigated in WMNs is that most of current works on QoS-aware protocols for WMNs are mainly based on a layered approach. This layered model led to the robust scalable protocols in the Internet and it has become the de facto architecture for wireless systems. However, the spatial reuse of the spectral frequency, the broadcast, unstable and error prone nature of the channel and different operational time scales for protocol layers, make the layered approach sub-optimum for the overall system performance of WMNs.

For instance, bad resource scheduling in MAC layer can lead to interference that affects the PHY layer performance due to reduced signal-to-interference-plus-noise-ratio (SINR) and ultimately deteriorates the overall network performance. Local capacity optimization with opportunistic scheduling techniques that exploit the multi-user diversity may increase the overall outgoing transceiver's throughput but they can also generate new bottlenecks in several routes in the network. Moreover, imprecise impact estimation of newly admitted applications on existing ones running in the network may jeopardize all ongoing QoS-aware services. 
As described above, limitations of layered architectures are stimulating the development of a new WMN cross-layer design. In a cross-layer paradigm, the joint optimization of control over two or more layers can yield significantly improved performance. In general, QoS implementations for WMNs can be classified based on network layered schemes (Gavrilovska \& Atanasovski, 2005).

Each layer has a set of mechanisms to provide quality level support for applications as following:

- MAC/LL Layer: Extensions of MAC mechanisms aim to provide QoS assurance in WMNs, such as IEEE 802.11e;

- Network Layer: Extensions of routing protocols and resource reservation schemes aim to provide QoS assurance in WMNs, such as The Optimized Link State Routing Protocol (OLSR) (Clausen \& Jacquet, 2006) routing protocol with QoE support as will be evaluated in Section 4 and presented in next section;

- Application Layer: Application layer QoS schemes aim to improve the distribution of multimedia content, by adapting sessions to the current network conditions;

- Cross-layer: Improves the overall system performance, by optimizing wireless resources and services based on information about more than one layer.

Recent advances in WMNs have introduced routing schemes as attractive solutions to provide end-to-end quality level control in such multi-hop scenarios. The number of hops was the first criteria adopted by traditional routing protocols. However, it is clear that these approaches are not suitable for multimedia applications, such as real-time video streaming, which require strict QoS guarantees.

Routing protocols need to be aware of the overhead caused by information exchange. This process generates traffic to gather routing information and therefore, it consumes bandwidth. In addition, mainly in the WMNs, the interference caused by the data and control frames transmitted also consumes bandwidth. Therefore, WMNs routing protocols need to minimize the amount of state information exchanged and also maximize the network throughput by using an appropriate selection process of the best path.

The best path can be defined by using a set of QoS and QoE parameters. There are three strategies to gather such information in WMNs:

- Proactive: each node maintains updated information about network topology in routing tables through the constant exchange of routing information. This information is transmitted by flooding on the network. When a source node needs to establish a route to the destination node, the route is selected by an appropriated algorithm based on the exchanged information;

- Reactive: protocols that belong to this category do not exchange routing information periodically, but gather routing information on-demand when it is required and therefore, a process of route discovery is started among involved nodes;

- Hybrid: this approach has advantages from both proactive and reactive protocols. Therefore, it reaches a good balance between proactive and reactive protocols. In addition, a hybrid protocol can be adaptive to a wide range of network characteristics (e.g., mobility and traffic patterns) and to optimize routing layer parameters for the different applications.

The implementation of routing schemes in WMNs is a hard task, but several solutions have been proposed. Among them, the OLSR protocol (Clausen \& Jacquet, 2006) is an adaptation of the traditional link-state algorithm for ad hoc networks. OLSR is a proactive protocol 
which uses a routing table obtained through the exchange of messages, between nodes, about the network conditions. A benefit of the OLSR protocol from the QoS perspective is its proactive nature that allows routes to be available before the source need to start a packet flow control to a destination. Another advantage of the OLSR protocol is that route computation is performed by using the knowledge about the entire network.

However, the hop count metric used by OLSR is unable to support QoS, because paths are selected based only on the number of hops (no well-know QoS metrics are used) along the session path. In this context, some extensions were developed for OLSR protocol, which are based on other link quality metrics. Among them, the OLSR Expected Transmission Count (ETX) and Minimum Delay (MD) are well-know metrics and will be presented below.

The OLSR extension based on ETX metric proposed in (De Couto et al., 2003) aims to find routes with the lowest expected number of transmissions that are necessary to ensure that a package can be delivered and has its arrival confirmed by the final destination. Other approach is the OLSR-MD (Cordeiro et al., 2007) that measures the link delay, calculating it through the Ad hoc Probe technique. Therefore, the calculation of the routing table can be based on the delay calculated to each neighboring node. Hence, in the OLSR-MD protocol the route selection between the current node and any other node in the network will have as criteria the lowest sum of the different transmission delays of all links along the path.

The OLSR-Dynamic Choice (OLSR-DC) extension (Gomes et al., 2008) aims to provide QoS support, giving different treatment to traffic from applications that use TCP and UDP, using the ETX metric for routing TCP packets and MD metric for routing UDP of packets. The protocol can also decouple the routing of TCP and UDP packets, this is achieved due to each packet be routed according to the metrics that best reflect their needs. This protocol was used as basis for the OLSR-FLC (Fuzzy Link Cost), since the proposed FLC is based on metrics that express the characteristics relevant to multimedia traffic. We can also configure FLC to route only UDP packets usually used for multimedia applications.

Comparing the previous solutions, OLSR-FLC seems to be the most suitable approach to guarantee the quality level support for multimedia applications in WMNs and will be explored and evaluated in this chapter. This novel cross-layer version uses a fuzzy logic to build a fuzzy system that aims to solve the problem of using multiple metrics for routing. The proposed fuzzy system has as base the values of the ETX and MD metrics collected from the network to define the FLC, which are used to route packets. TCP packets are still routed based on the ETX metric, as occurs with the OLSR-DC protocol. A detailed description of the OLSR-FLC can be found in (Gomes et al., 2009).

A major challenge regarding QoS-aware systems, including WMN routing schemes, is the lack of solutions to assure quality level control for applications according to the user's perception. Traditional measurement schemes on the network can be used to estimate the impact of the quality of a media, such as video, but do not represent the entire set of metrics that will enable the management end-to-end quality-focused user's experience. Network statistics alone do not represent the perception of the user (Siller \& Woods, 2003).

Current techniques that aim to maximize the quality level of multimedia services on a network are centralized in the aspects of QoS-based schemes that define a set of control operations and measurement, at the network level and packages to ensure the distribution of multimedia content in wired and wireless, with an acceptable level of quality (Zapater \& Bressan, 2007). However, existing QoS metrics such as package loss rate, delay and 
throughput, are typically used to indicate the impact of the quality of a video (or any media) from the viewpoint of the network, but not reflects the situation experienced by the user. Consequently, these QoS parameters fail to capture the subjective aspects associated with human perception.

In order to overcome the current limitations of networks in their schemes of QoS for multimedia applications, considering the aspects of human perception and subjectivity related to the approach of QoE has been introduced (Takahashi et al., 2008), as characteristics of feelings, perceptions, views of users and how they interact with their environments and can be enjoyable and fun or annoying and frustrating (Patrick et al., 2004).

\subsection{Quality of experience Issues}

QoE issues have been creating a new assessment and management paradigm in multimedia systems and gaining a special attention in WMNs. QoE metrics have considered important metrics to measure the quality level of multimedia content based on the user's perspective (Rowe \& Jain, 2005) (De Vleeschauwer et al., 2008). QoE approaches aim to overcome the limitations of current QoS-aware schemes regarding human perception and subjectiverelated aspects (Jain, 2004) (Klein, 2007).

The emerging of QoE issues required the inclusion of a new user-level (abstraction) layer on Open Systems Interconnection (OSI) and Internet architectures (Siller \& Woods, 2003). This layer can be seen as an extension of the application layer with user's perception (Bauer \& Patrick, 2004). Therefore, the results of QoE procedures can be used as an extension to the traditional QoS in the sense that QoE provides information regarding the delivered multimedia service from the user's point of view. Hence, QoE procedures can be explored to improve the accuracy of QoS control plane operations and to ensure smooth transmission of audio and video over WMNs.

It is important to highlight that QoE results are widely dependent on subjective aspects related with human perception, as well as, user's location, screen size, hardware and applications (Valerdi et al., 2009) (Bhatti et al., 2000). For instance, video sequences with different complexities, motions and frame rates will produce different QoE results (Greengrass et al., 2009).

QoE measurement operations can be used as an indicator of how a networking environment meets the end-user needs. The QoE applicability scenarios, requirements, evaluations and assessment methodologies in multimedia systems have been investigated by several researchers and working groups, such as International Telecommunication Union Telecommunication Standardization Sector (ITU-T) (ITU-T, 2010), Video Quality Experts Group (VQEG) (VQEG, 2010) and European Technical Committee for Speech, Transmission, Planning, and Quality of Service (ETSI STQ) (ETSI, 2010).

Advances in QoE-aware systems will allow the deployment of new QoS/QoE-sensitive services as well as provide new paradigms for the creation of new protocols, assessment solutions, objective and subjective metrics, routing approaches and overlay networks, such as the deployment of QoE routing schemes and user-aware packet controllers. Nowadays, QoE operations are not fully implemented in end-to-end networking systems due to the high CPU and memory consumption required by current QoE schemes, as well as to the lack of accuracy of in-service quality assessment methods. Usually, only QoE out-service measurement procedures are accomplished to evaluate the quality level of multimedia services WMNs and other systems. 
Regarding QoE assessment issues, matching the multimedia quality level by computerized measurement is a research challenge and needs to take as input many factors related with the user's perception. Multimedia quality evaluation approaches are classified into two main orthogonal criterions as described in the remainder subsections and presented in Figure 2: (i) the amount of the reference information required to assess the quality and (ii) the measured features based on objectivity/subjectivity (i.e. the way the quality is expressed).

\subsubsection{Classification based on objectivity and subjectivity}

In general, there are main methods to assess the quality level of multimedia contents, namely objectivity, subjectivity and hybrid. The output of these schemes is useful for QoEaware billing/accounting procedures, assessment solutions and management issues.

Subjective metrics assess how audio and/or video streams are perceived by users (Kishigami, 2007), i.e., what is their opinion on the quality of particular audio/video sequences, as described in ITU-T recommendation BT 500 (ITU-R, 1995). The most popular subjective metric is called Mean Option Score (MOS). The quality level of a video (or audio) sequence based on MOS model is rated on a scale of 1 to 5 , where 5 is the best possible score as presented in Table 1 .

The MOS values are achieved based on subjective tests and methodologies performed with a set of viewers. For instance, the Single Stimulus Continuous Quality Evaluation (SSCQE) test allows viewers to dynamically rate the quality of an arbitrarily long video sequence using a slider mechanism with an associated quality scale. The drawback of subjective metrics is the fact that they are neither practical nor scalable for real-time multimedia environments. Other approaches are Double Stimulus Impairment Scale (DSIS), Double Stimulus Continuous Quality Scale (DSCQS), Single Stimulus Continuous Quality Evaluation (SSCQE), Simultaneous Double Stimulus for Continuous Evaluation (SDSCE) and Stimulus Comparison Adjectival Categorical Judgment (SCACJ) (Bocca-Rodríguez et al., 2007).

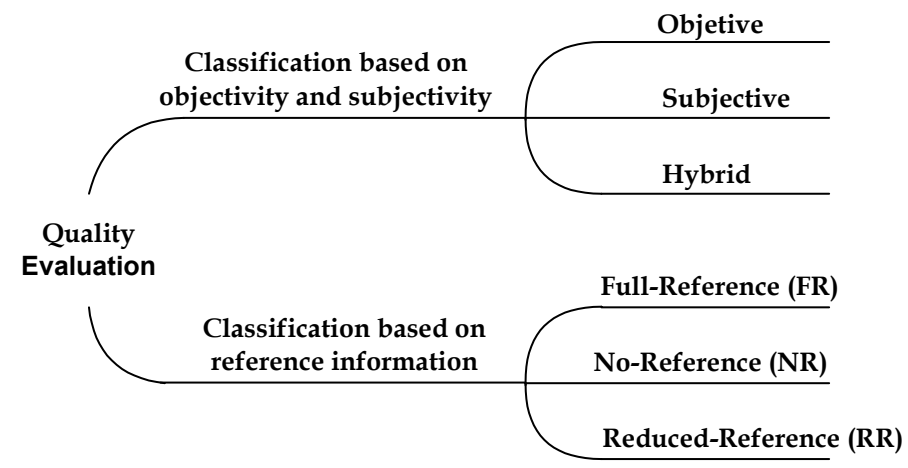

Fig. 2. Quality evaluation (Romaniak et al. 2008)

Several objective QoE metrics have been developed to estimate/predict (based on mathematical models) the quality level of multimedia services according to the user's perception. Among them, the Peak Signal to Noise Ratio (PSNR) is a traditional objective metric used to measure, in decibels, the video quality level based on original and processed 


\begin{tabular}{|c|l|l|}
\hline MOS & Quality & Impairment \\
\hline 5 & Excellent & Imperceptible \\
\hline 4 & Good & Perceptible but not annoying \\
\hline 3 & Fair & Slightly annoying \\
\hline 2 & Poor & Annoying \\
\hline 1 & Bad & Very annoying \\
\hline
\end{tabular}

Table 1. Mean Option Score

video sequences. Typical values for the PSNR in lossy videos are between $30 \mathrm{~dB}$ and $50 \mathrm{~dB}$, where higher is better. The PSNR of a video is defined through the Mean Square Error (MSE) metric. Considering the luminance $(\mathrm{Y})$ of the processed and original frames and assuming frames with MxN pixels, the MSE is obtained using the Equation 1.

$$
M S E=\frac{1}{M x N} \sum_{i=0}^{M-1} \sum_{j=0}^{N-1}\left\|Y_{S}(i, j)-Y d(i, j)\right\|^{2}
$$

In Equation 1, while $Y_{s}(i, j)$ designates the pixel in the position $(i, j)$ of the original frame, the $\mathrm{Yd}(\mathrm{i}, \mathrm{j})$ represents the pixel located in the position $(i, j)$ of the processed frame. Based on the MSE definition and on 8bits/sample, the PSNR, in a logarithmic scale, is achieved using the Equation 2.

$$
P S N R=20 \log _{10}\left(\frac{255}{\sqrt{\frac{1}{M x N} \sum_{i=o}^{M-1} \sum_{j=0}^{N-1}\|Y s(i, j)-Y d(i, j)\|^{2}}}\right)
$$

The MSE and PSNR metrics only provide an indication of the difference between the received frame and a reference signal, and do not consider any other important aspects which can strongly influence the video quality level, such as Human Visual System (HVS) characteristics (a detailed analysis of HVS can be found in (Wang et al., 2004).

The Structural Similarity Index Metric (SSIM) improves the traditional PSNR and MSE, which are inconsistent with HVS characteristics, such as human eye perception (Wang et al., 2004). The SSIM metric is based on frame-to-frame measuring of three components (luminance similarity, contrast similarity and structural similarity) and combining them into a single value, called index. The SSIM index is a decimal value between 0 and 1 , where 0 means no correlation with the original image, and 1 means the exact same image.

The Video Quality Metric (VQM) method defines a set of computational models that also have been shown to be superior to traditional PSNR and MSE metrics (Revés et al., 2006). The VQM method takes as input the original video and the processed video and verifies the multimedia quality level based on human eye perception and subjectivity aspects, including blurring, global noise, block distortion and color distortion. The VQM evaluation results vary from 0 to 5 values, where 0 is the best possible score. 
The Moving Picture Quality Metric (MPQM) evaluates the video quality using HVS modeling characteristics (Lambrecht, \& Verscheure, 1996). The input to the MPQM metric is an original video sequence and a distorted version of it. The distortion is first computed as the difference between the original and the distorted sequences. The original and the error sequences are then decomposed into perceptual channels segmented using uniform areas, textures and contours classification.

\subsubsection{Reference-based classification}

Three different approaches are used to classify video quality assessment methods, based on reference-related video procedures, namely Full Reference (FR), Reduced Reference (RR) and No Reference (NR) (Engelke \& Zepernick, 2007) (Garcia et al., 2009).

The FR approach assumes unlimited access to the original multimedia sequence. This approach uses the video reference to predict the quality level (degradation) of the processed video, by comparing the difference of every pixel in each image of the distorted video with its corresponding pixel in the original video. As consequence, it provides, in general, superior quality assessment performance. The FR method is difficult to implement in realtime networking systems (QoE-aware equipment/monitoring agent) because it always requires the original sequence during the evaluation process (common for offline experiments). Examples of metrics based on an FR approach are PSNR, SSIM and MPQM.

For in-service video quality measurements, RR and NR approaches are generally more suitable. The RR approach differs from the FR approach only selected multimedia parameters (or characteristics) are required during quality evaluation process, such as motion information. The set of reference parameters can be transmitted piggy-backed with the multimedia flow or by using a secondary channel. The objective of RR is to be as accurate as the full reference model, although using less network and processing resources. An example of an RR scheme is Video Quality Model (VQM), developed by the National Telecommunications and Information Administrative (NTIA) and reported in (Pinson \& Wolf , 2004).

The NR approach tries to assess the quality of a distorted multimedia service without any reference to the original content. This approach is usually used when the coding method is known. NR-based metrics can be used in in-service network monitoring/diagnostic operations, when the original multimedia sequence is not available. The drawbacks of NR metric are the following: (i) low correlation with MOS; (ii) high CPU and memory consumption; (iii) time limitation. An example of NR schemes is the V-Factor model (VFactor 2010) that outputs MOS.

\section{Cross-layer design}

The methodology of layered protocol design has been applied for decades in different types of network, for instance, OSI and Internet architectures. In this model, protocols, services and applications are designed without being constrained by each other. Many advantages such as scalability of network size, portability of protocols in different layers, flexibility in protocol design, and so on can be easily obtained in layered architectures. However, advances in emerging networks and heterogeneous systems are changing the traditional layered model.

There are many reasons behind the improvement of the layered design as follows: (i) the requirement of service quality is ever-increasing; (ii) the network heterogeneity is much 
higher than years ago; (iii) the conventional layered architecture is effective for integrating them into the same network, but tile performance is not optimized; (iv) many networks today, especially wireless networks have no dedicated links between nodes. In a wireless network, transmission between two nodes also interferes with other nodes in the neighborhood. Thus, the meaning of "link" pertained to a conventional wired network does not exist anymore. The capacity of a link is variable and can be fully cross-related with other links. Such inter-dependence in fact breaks the transparency between different protocol layers, where a multi-hop network, such as IEEE 802.11s, is concerned, this problem becomes much more obvious.

QoS and QoE support for multimedia application is a good example to explain the need of a cross-layer design, where the end-to-end quality level support over emerging systems involves the cooperation of three layers, namely physical, MAC and network. Therefore, a cross-layer paradigm must be applied to allow a tight communication between layers and improve the system performance (Kozat et al., 2004).

Several networking proposals have created to explore the benefits of cross-layer architectures to increase the network performance (Kawadia \& Kumar, 2005) (Bhatia \& Kodialam, 2004) (Chiang, 2004) (Kozat et al., 2004). The design of cross-layer models can be done by using two main approaches as presented in Figure 3, namely loosely coupled crosslayer design and tightly coupled cross-layer design.

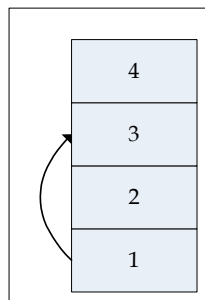

(a)

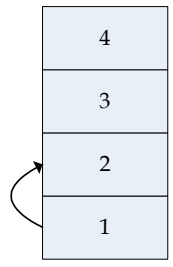

(b)

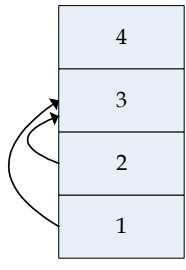

(c)

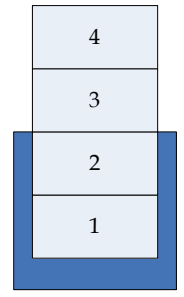

(d)

Fig. 3. Loosely coupled cross-layer design and rightly coupled cross-layer design

In the loosely coupled cross-layer design, the optimization is carried out without crossing layers, but focusing on one protocol layer. In order to improve tine performance of this protocol layer, parameters in other protocol layers are taken into account. Thus, information to one layer must be passed to another layer. Typically, parameters in the lower protocol layers are reported to higher layers. For example (Figure 3a), the packet loss rate in the MAC layer, or channel condition in the physical layer can be reported to the transport layer so that a TCP protocol is able to differentiate congestion from packet loss. As another example (Figure $3 b$ ), the physical layer can report the link quality to a routing protocol as an additional performance metric for the routing algorithms.

It should be noted that information from multiple layers can be used on another layer to perform cross-layer design (Figure 3c). There are two different ways of utilizing cross-layer information. The first one is the simplest case of cross-layer design, in which the information in other layers works just as one of the parameters needed by the algorithm in a protocol layer. The performance of this algorithm is improved because a better (more accurate or reliable) parameter is used, but the algorithm itself does not need a modification. For 
example, the physical layer can inform the TCP layer of the channel quality so that TCP can differentiate real congestion from channel quality degradation, and thus carry out congestion control more intelligently. In the second method, based on the information from other layers, the algorithms of a protocol have to be changed. For example, if a MAC protocol can provide a routine protocol information about its performance, the routing can perform multipath routing to utilize spatial diversity. However, the change from single-path routing to multipath routing needs a significant modification to the routing protocol rather than just parameter adaptation.

In the tightly coupled cross-layer design (Figure 3d), merely information sharing between layers is not enough. In this scheme, the algorithms in different layers are optimized together as one optimization problem. For example (Figure 3d), for MAC and routing protocols in a multichannel TDMA WMN, timeslots, channels, and routing path can be determined by one single algorithm. Using optimization across layers, it can be expected that much better performance improvement can be achieved by the tightly coupled crosslayer design than the loosely coupled scheme. However, the advantage of the loosely coupled design is that it does not totally abandon the transparency between protocol layers. An extreme case of tightly coupled cross-layer design is to merge different protocol layers into one layer. According to the concept of "layering as optimization decomposition", this kind of design tries to improve network performance by re-layering the existing protocol stack. Merging multiple protocol layers into one layer keeps the advantage of tightly coupled cross-layer design. Furthermore, it can also eliminate the overhead in cross-layer information passing and is a trend in WMNs.

Interestingly, merging multiple protocol layers is not just a theoretical concept, but has been seriously considered in real practice. For example, in the IEEE 802.11s, the routing protocol is being developed as one of the critical modules in the MAC layer. Such a merging between routing and MAC layers provides great potential for carrying out optimization across MAC and routing, based on the same algorithm. Recent advances in wireless optimization are attracting researchers and industry to study cross-layer issues for future networks (Chen et al., 2007). Other cross-layer implementations supporting QoS in wireless system, as well as, load balance techniques can be found in (Pahalawatta et al., 2007), (Wu et al., 2007) and (Villalon et al., 2007).

It is clear that the cross-layer schemes will be predominant in WMNs, where new quality level mechanisms will be designed and implemented to increase the satisfaction of costumers and optimize the usage of network resources, such as routing protocols.

\section{Performance evolution}

As presented before, routing protocols based on QoE aims to optimize the usage of network resources, the system performance and the quality level of multimedia applications in WMNs. Novel QoE-aware cross-layer solutions will be essential for the success of next generation wireless system. In order to show the impact of this kind of solution on the user's experience, this section presents the behavior of the OLSR-FLC protocol in WMNs, as well as to show the benefits, comparing it with the main well-known extensions of the OLSR protocol, OLSR-ETX, OLSR-MD, OLSR-DC.

This cross-layer OLSR extension is based on the dynamic choice of link quality metrics and in a FLC to decide on paths for multimedia packages. We analyzed the performance of the proposal through simulations on Network Simulator (NS-2) (Fall \& Varadhan, 2010), using the scenario shown in Figure 4, which represents the WMN backbone partiality deployed in the Federal University of Para (UFPA) campus. 
Table 2 describes the simulation parameters, which try to bring the simulation as close as possible to the considered real network scenario, representing the characteristics of the region and the used equipments. Path Loss Exponent and Shadowing Deviation parameters were used according to the measurements presented in (Moreira et al., 2008). The routers' carrier sense threshold and transmit power parameters were based on the IEEE 802.11 standard. The other values were used to represent the antennas and the routers used in the WMN at UFPA.

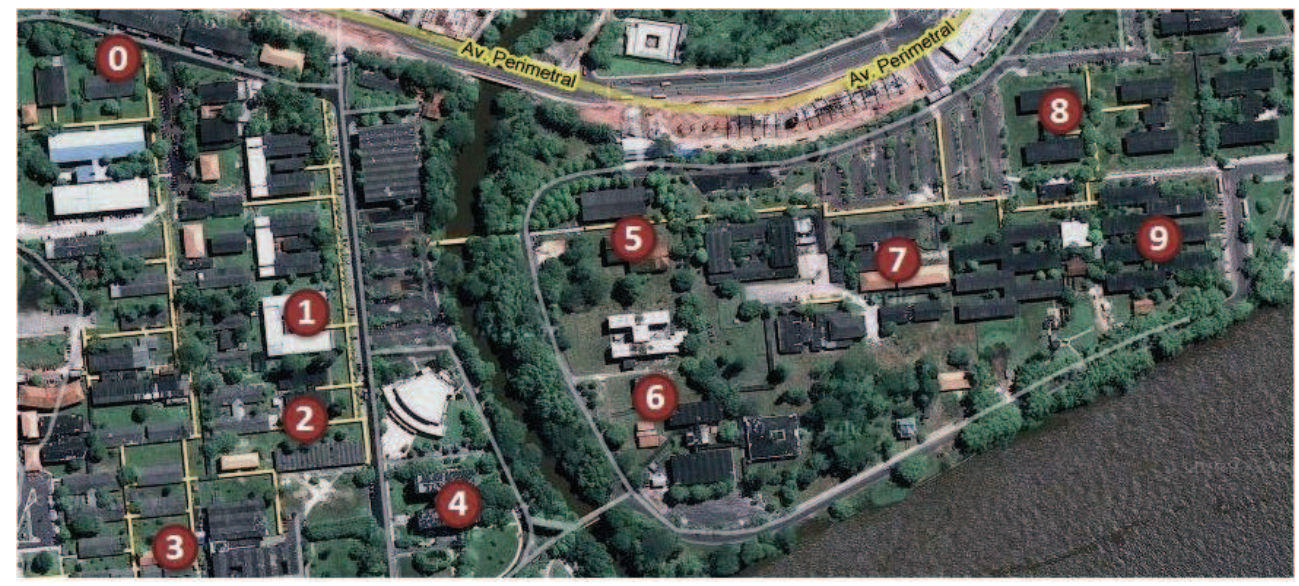

Fig. 4. Simulated scenario

\begin{tabular}{|l|l|}
\hline Parameter & Value \\
\hline Standard IEEE & $802.11 \mathrm{~g}$ \\
\hline Propagation Model & Shadowing \\
\hline Antenna & Omnidirectional 18dB \\
\hline Router's Carrier Sense Threshold & $-76 \mathrm{dBm}$ \\
\hline Router's Transmit Power & $-80 \mathrm{dBm}$ \\
\hline Tansmission Power & $17 \mathrm{dBm}$ (WRT54G) \\
\hline Frequency & $2.422 \mathrm{GHz}($ Channel 3) \\
\hline Path Loss Exponent & 1.59 \\
\hline Shadowing Deviation & $5.4 \mathrm{~dB}$ \\
\hline
\end{tabular}

Table 2. Simulation Parameters

Twenty simulations were performed using different seeds for each protocol: OLSR, OLSRETX, OLSR-MD, OLSR-DC, and OLSR-FLC. Table 3 shows the flow configuration used. All simulations were run for 50 seconds.

The configuration of flows aims to balance the flow over the MWN topology and to create a higher competition scenario, between data, audio, and video traffics. Hence, it brings the simulation to a common situation in WMNs, i.e., competition among all kind of flows where each flow has its own characteristics and requirements. 
The simulation experiments comprised 3 VoIP (Voice over IP) calls, that on simulations are characterized by two flows, i.e. 6 UDP flows, 5 TCP-Reno flows and 3 video traffic. The video traffics were evaluated from the experience that the user obtained, through the QoE evaluation metric. The UDP flows have a bit rate of $8 \mathrm{~Kb} / \mathrm{s}$ and 40 bytes (RTP + UDP + Payload) of packet size, in order to represent the G.729 codec (Balam \& Gibson, 2007). The TCP flows were characterized as FTP applications, following the Pareto model with a rate of 200k, 210 bytes of packet size and 500 ms burst duration.

\begin{tabular}{|c|c|c|c|c|l|}
\hline Flow & Source & Destiny & Begin & End & Traffic \\
\hline 1 & 1 & 8 & 10 & 40 & TCP - Reno \\
\hline 2 & 9 & 2 & 11 & 41 & TCP Reno \\
\hline 3 & 7 & 4 & 12 & 42 & TCP Reno \\
\hline 4 & 5 & 0 & 13 & 43 & TCP Reno \\
\hline 5 & 6 & 4 & 14 & 44 & TCP Reno \\
\hline 6 & 0 & 5 & 10 & 45 & Video Paris \\
\hline 7 & 3 & 6 & 14 & 29 & Video Foreman \\
\hline 8 & 3 & 6 & 30 & 45 & Video News \\
\hline 9 & 2 & 9 & 6 & 46 & UDP - CBR \\
\hline 10 & 9 & 2 & 6 & 46 & UDP - CBR \\
\hline 11 & 1 & 8 & 7 & 47 & UDP - CBR \\
\hline 12 & 8 & 1 & 7 & 47 & UDP - CBR \\
\hline 13 & 4 & 7 & 8 & 48 & UDP - CBR \\
\hline 14 & 7 & 4 & 8 & 48 & UDP - CBR \\
\hline
\end{tabular}

Table 3. Flow Configuration

The video traffic was simulated through the Evalvid tool (Evalvid, 2010) that allows the control of the video quality in a simulation environment. Well-know real videos sequences were used, namely "Paris", "Foreman" and "News". These videos have frames in YUV format, which are compressed by MPEG-4 codec and sent at a rate of 30 frame/s. Each frame was fragmented into blocks of 1024 bytes where the packet has a size of 1052 bytes.

Well-know objective and subjective QoE metrics are used in the experiments, following the tests proposed by ITU-R (ITU-R, 1995). The subjective QoE metrics evaluate the quality of multimedia applications based on the receiver's opinion, where the MOS was used. The videos were analyzed using the MSU Video Quality Measurement Tool Software (MSU, 2010). The value of PSNR is expressed in $\mathrm{dB}$ (decibels). For a video to be considered with good quality it should have an average PSNR of at least $30 \mathrm{~dB}$.

The following tables and figures will demonstrate the simulation results of real video sequences collected from all protocols and based on QoS metrics. The tables show the average values, the highest value, the lowest value and standard deviation values for each 
protocol. Tables 4 and 5 show the values for the video "Foreman", where Table 4 presents the values of VQM and SSIM metrics and Table 5 the values of PSNR and MOS metrics. The same results are illustrated in Figure 5, 6 and 7 respectively.

\begin{tabular}{|l|c|c|c|c|c|c|c|c|}
\hline Foreman & & & VQM & & & & SSIM & \\
\hline & Higher & Lower & Average & $\begin{array}{c}\text { Standard } \\
\text { Deviation }\end{array}$ & Higher & Lower & Average & $\begin{array}{c}\text { Standard } \\
\text { Deviation }\end{array}$ \\
\hline OLSR & 5 & 4,8 & 4,96 & 0,07 & 0,7 & 0,5 & 0,58 & 0,07 \\
\hline OLSR-MD & 5 & 4 & 4,72 & 0,32 & 0,71 & 0,62 & 0,66 & 0,04 \\
\hline OLSR-ETX & 5 & 4,5 & 4,86 & 0,23 & 0,77 & 0,5 & 0,61 & 0,08 \\
\hline OLSR-DC & 5 & 2,3 & 4,5 & 0,83 & 0,87 & 0,61 & 0,67 & 0,08 \\
\hline OLSR-FLC & 4,8 & 2,4 & 4,27 & 0,71 & 0,83 & 0,68 & 0,73 & 0,04 \\
\hline
\end{tabular}

Table 4. VQM and SSIM Values of Video Foreman

\begin{tabular}{|l|c|c|c|c|c|}
\hline \multicolumn{1}{|c|}{ Foreman } & Higher & Lower & Average & $\begin{array}{c}\text { Standard } \\
\text { Deviation }\end{array}$ & \\
\hline OLSR & 18 & 14 & 15,8 & 1,62 & Bad \\
\hline OLSR-MD & 23 & 16 & 19,1 & 2,26 & Bad \\
\hline OLSR-ETX & 20 & 13 & 17,4 & 2,63 & Bad \\
\hline OLSR-DC & 25 & 17 & 19,3 & 2,58 & Bad \\
\hline OLSR-FLC & 25 & 22 & 22,9 & 0,88 & Poor \\
\hline
\end{tabular}

Table 5. PSNR and MOS Values of Video Foreman

The transmission of the "Foreman" video, flow 7, begins after all the flows start their transmissions, and it starts in a moment of convergence of the protocols, which results in a high difficulty transmission with network congestion. These facts become clear from the data shown in the tables for the "Foreman" Video. Therefore, OLSR-FLC has the best values of QoE metrics, and is the only one which achieves the "Poor" quality while the other protocols obtain a quality considered "Bad", although the protocols OLSR-DC and OLSRMD have values close to being considered as "Poor".

The Tables 6 and 7 show the values for the video "News", where Table 6 presents the values of VQM and SSIM values and Table 7 the values of PSNR and MOS metrics. The "News" video, flow 8, has the same destination and source as flow 7, "Foreman" video, however, it starts at a different time of the simulation. At this moment the protocols had already passed by the period of convergence, allowing a better choice of routes. 


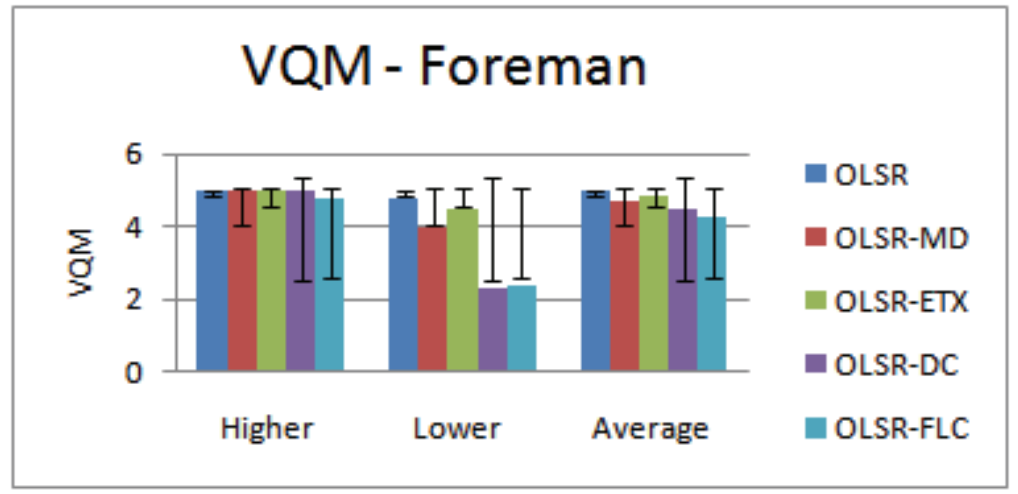

Fig. 5. VQM results of the Foreman video sequence for all protocols

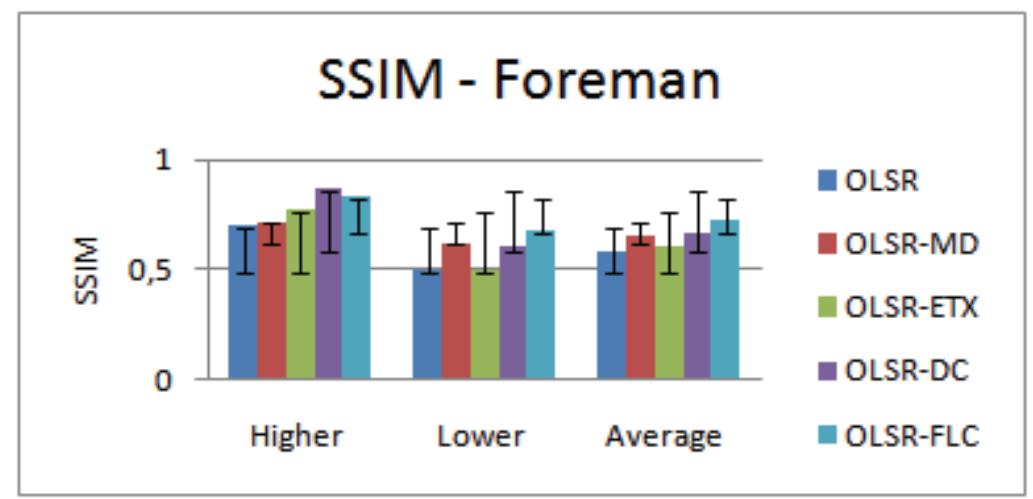

Fig. 6. SSIM results of the Foreman video sequence for all protocols



Fig. 7. PSNR results of the Foreman video sequence for all protocols 


\begin{tabular}{|l|c|c|c|c|c|c|c|c|}
\hline \multicolumn{1}{|c|}{ News } & Higher & Lower & Average & $\begin{array}{c}\text { Standard } \\
\text { Deviation }\end{array}$ & Higher & Lower & Average & $\begin{array}{c}\text { Standard } \\
\text { Deviation }\end{array}$ \\
\hline OLSR & 5 & 1,7 & 4,43 & 1,08 & 0,86 & 0,71 & 0,79 & 0,05 \\
\hline OLSR-MD & 5 & 2,2 & 3,71 & 1,05 & 0,95 & 0,83 & 0,89 & 0,04 \\
\hline OLSR-ETX & 5 & 2,2 & 4,09 & 1,01 & 0,89 & 0,78 & 0,85 & 0,04 \\
\hline OLSR-DC & 4,9 & 1,4 & 3,44 & 1,17 & 0,97 & 0,84 & 0,89 & 0,05 \\
\hline OLSR-FLC & 4 & 0,5 & 3,08 & 1,01 & 0,98 & 0,85 & 0,91 & 0,04 \\
\hline
\end{tabular}

Table 6. VQM and SSIM Values of Video News

\begin{tabular}{|l|c|c|c|c|c|}
\hline \multicolumn{1}{|c|}{ News } & Higher & Lower & Average & $\begin{array}{c}\text { Standard } \\
\text { Deviation }\end{array}$ & MOS \\
\hline OLSR & 25 & 17 & 19,7 & 2,91 & Bad \\
\hline OLSR-MD & 27 & 19 & 22,5 & 3,21 & Poor \\
\hline OLSR-ETX & 29 & 17 & 20,7 & 4,22 & Poor \\
\hline OLSR-DC & 27 & 19 & 23,6 & 2,84 & Poor \\
\hline OLSR-FLC & 44 & 20 & 25,7 & 7,01 & Regular \\
\hline
\end{tabular}

Table 7. PSNR and MOS Values of Video News

We note this by comparing the data of "News" video with the data of "Foreman" video, where the "News" video has better values of QoE metrics. Again OLSR-FLC achieves the best video quality, having a quality considered "Regular", while the other protocols obtain qualities ranging from "Poor" to "Bad". Despite of having a better video quality, OLSR-FLC has a high standard deviation, showing a degree of instability in the quality of the transmitted videos, obtaining values better and of similar quality to the other protocols.

The Figure 8 and 9 show the values for the video "Paris", where Table 8 presents the values of VQM and SSIM values and Table 9 the values of PSNR and MOS metrics. Since the "Paris" video, flow 6, is longer than the other videos, it is transmitted during almost the entire simulation, this means that the transmission has a hard time during the convergence of the protocols at the beginning of its transmission, but most of the transmission occurs after the convergence period.

Unlike the other video transmissions, flows 7 and 8, the nodes involved in flow 6 have a clear line of sight, however, with a higher distance between the nodes. This makes that the use of a single hop increase the chance of packet loss, as well as, the use of multiples hops increase the end-to-end delay of the package. Within this reality, the usage of a single metric turns out to be insufficient to find the most appropriate route, because a good video transmission depends not only on small losses, but also on a small delay and jitter. 


\begin{tabular}{|l|c|c|c|c|c|c|c|c|}
\hline \multicolumn{1}{|c|}{ Paris } & Higher & Lower & Average & $\begin{array}{c}\text { Standard } \\
\text { Deviation }\end{array}$ & Higher & Lower & Average & $\begin{array}{c}\text { Standard } \\
\text { Deviation }\end{array}$ \\
\hline OLSR & 5 & 4,4 & 4,93 & 0,19 & 0,75 & 0,63 & 0,69 & 0,04 \\
\hline OLSR-MD & 3,8 & 3 & 3,35 & 0,31 & 0,9 & 0,83 & 0,86 & 0,02 \\
\hline $\begin{array}{l}\text { OLSR- } \\
\text { ETX }\end{array}$ & 4,9 & 3 & 4,09 & 0,59 & 0,83 & 0,73 & 0,79 & 0,04 \\
\hline OLSR-DC & 3,5 & 2,3 & 2,94 & 0,41 & 0,93 & 0,87 & 0,88 & 0,02 \\
\hline $\begin{array}{l}\text { OLSR- } \\
\text { FLC }\end{array}$ & 3,1 & 2,3 & 2,75 & 0,32 & 0,93 & 0,87 & 0,91 & 0,02 \\
\hline
\end{tabular}

Table 8. VQM and SSIM Values of Video Paris

\begin{tabular}{|l|c|c|c|c|c|}
\hline \multicolumn{1}{|c|}{ Paris } & Higher & Lower & Average & $\begin{array}{c}\text { Standard } \\
\text { Deviation }\end{array}$ & MOS \\
\hline OLSR & 17 & 14 & 15,2 & 1,23 & Bad \\
\hline OLSR-MD & 27 & 23 & 24,8 & 1,14 & Poor \\
\hline OLSR-ETX & 23 & 20 & 21,4 & 1,17 & Poor \\
\hline OLSR-DC & 29 & 23 & 26,5 & 2,07 & Regular \\
\hline OLSR-FLC & 31 & 25 & 29,2 & 2,15 & Regular \\
\hline
\end{tabular}

Table 9. PSNR and MOS Values of Video Paris

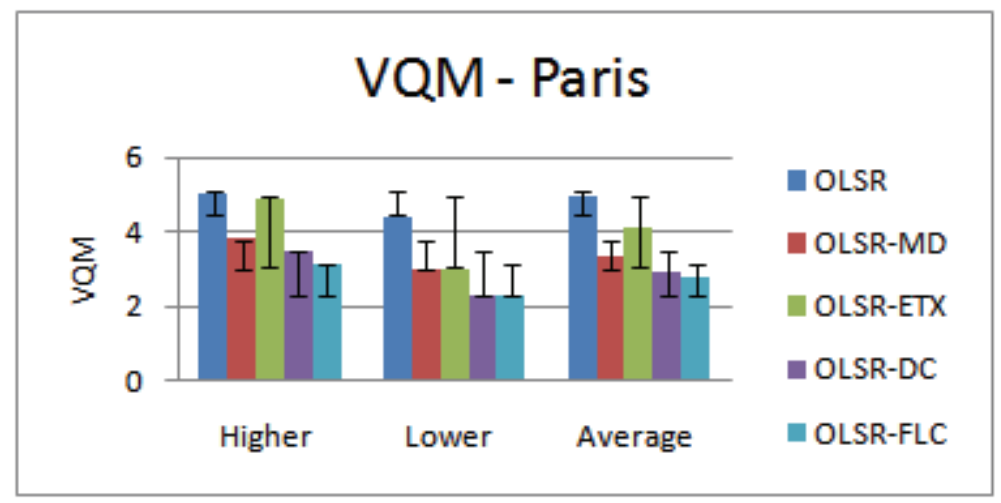

Fig. 8. VQM values for Paris video sequence

Therefore, we note that OLSR-FLC can adapt to this reality of multiple requirements, which is visible in the tables for the "Paris" video. The OLSR-FLC protocol, as well as, the OLSR- 
DC protocol, have a video quality considered "regular", however OLSR-FLC reaches values close to "Good" quality level. In other words, because it is based on the OLSR-DC protocol, the OLSR-FLC protocol can better distribute the traffic, but it uses a fuzzy link cost, based on delay and quality of links. This enables the protocol to obtain a better video quality, against the protocols that use only one metric for routing.

In some of the existing works, as in (Moreira et al., 2008), (Gomes et al., 2008) and (Gomes et al., 2009a), the evaluation of the protocols occurred in scenarios of competition, however, the competition present in these works is small, when compared with the scenario used in this work, due to the quantity of flows used. This evaluation shows that only one metric for routing data and multimedia traffics may not be sufficient to reach acceptable QoS and QoE levels to answer the needs of the traffics, since each one has different requirements.

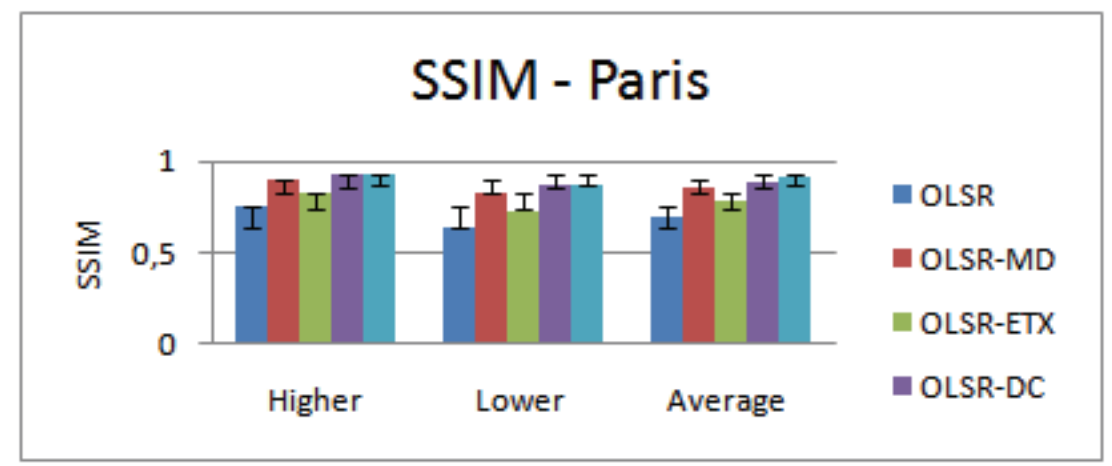

Fig. 9. SSIM values for Paris video sequence

\section{Final considerations}

Currently, wireless systems, multimedia distribution and quality level control continue to be strong research areas. Trends in these areas are expected to continue with various challenges emerging as a result of new services, protocols, wireless mesh networks, cross-layer schemes, emerging portable devices, changing user and terminal requirements. It is clear that recent advances on QoS/QoE have been allowed the creation of a new ubiquitous wireless multimedia approach in the Internet.

WMN routing solutions with QoE support will be essential for the success of multimedia communications, where users will be more satisfied with the received content and mobile operators will be able to increase their billing with the new attractions to clients and the operational costs reduction. However, one of the main challenges is to develop and implement new in-service routing QoE solutions in WMNs.

This chapter was intended to highlight important topics in WMNs, QoS, QoE, routing and cross-layer areas that need attention to address some of the most pressing challenges associated with them. We focus on four key areas, where the first one was on QoS, the second one on QoE, the third one on routing and the last on cross-layer issues. Furthermore, in order to demonstrate the behavior of an implementation of a QoE-aware cross-layer routing in WMNs, simulations were carried out. The results show the benefits of the proposed scheme on the user's perspective, by using well-know QoE metrics. 
We hope that this work will help improve our understanding of the issues and challenges that lie ahead in wireless mesh networks and QoS/QoE issues will serve as a catalyst for designers, engineers, and researchers to seek innovative solutions to address and solve those challenges.

\section{Acknowledgements}

Eduardo Cerqueira was supported by CNPq 476202/2009-4 \& 557.128/2009-9, PROPESP UFPA and FAPESPA 5183.UNI319.4107.07072009 - 5467.UNI317.1279.31082009. We would like also thanks André Riker and Patricia Araujo de Oliveira for their value contributions for this chapter.

\section{References}

802.11s (2010). IEEE Mesh Networking, Task Group S. Status of Project IEEE 802.11s, accessed in March 2010, of http://www.ieee802.org/11/Reports/tgs_update.htm

802.16 (2010). IEEE Standard. Working Group on Broadband Wireless Access, accessed in March 2010, of http:/ / grouper.ieee.org/groups/802/16/

Akyildiz, I. \& Wang, X. (2009). Wireless Mesh Networks (Advanced Texts in Communications and Networking), Wiley

Balam, J. \& Gibson, J (2007). Multiple descriptions and path diversity for voice communications over wireless mesh networks. IEEE Transactions on Multimedia, pages 1073-1088, August 2007.

Bauer, Ben; Patrick, Andrew S. (2004). A Human Factors Extension to the Seven-Layer OSI Reference Model, January 2004, accessed in April 2010, of http://www.andrewpatrick.ca/OSI/10layer.html

Bhatia, R. \& Kodialam, M. (2004). On power efficient communication over multi-hop wireless networks: joint routing, scheduling and power control, Twenty-third AnnualJoint Conference of the IEEE Computer and Communications Societies, Vol.2, pp. 1457- 1466, March 2004, INFOCOM 2004

Bhatti, Nina; Bouch, Anna; Kuchinsky, Allan (2000). Integrating user-perceived quality into Web server design. Proceedings of the 9th international World Wide Web conference on Computer networks: the international journal of computer and telecommunications networking. Amsterdam, Vol. 33, No. 1-6, (June 2000) page numbers (1-16)

Bocca-Rodríguez, Pablo; Cancela, Héctor; Rubino, Gerardo (2007). Video Quality Assurance in Multi-Source Streaming Techniques, Proceedings of the 4th international IFIP/ACM Latin American conference on Networking, pp. 83-93, Applications, Technologies, Architectures, and Protocols for Computer Communication, 2007, San José

Bok-Nyong Park et al. (2006). QoS-driven wireless broadband home networking based on multihop wireless mesh networks. Consumer Electronics, IEEE Transactions on, Vol. 52, No. 4, (November 2006) page numbers (1220-1228)

Chen, B.; Lee, M.J. \& Sun, Y. (2007). A Framework for Crosslayer Optimization from Physical Layer to Routing Layer on Wireless Ad Hoc Networks, Global Telecommunications Conference, pp. 3678-3683, November 2007, GLOBECOM '07

Chiang, M (2004). To layer or not to layer: balancing transport and physical layers in wireless multihop networks, Twenty-third Annualjoint Conference of the IEEE Computer and Communications Societies, Vol.4, pp. 2525- 2536, March 2004, INFOCOM 2004 
Chiti, F.; Fantacci, R.; Maccari, L.; Marabissi, D. \& Tarchi, D. (2008). A broadband wireless communications system for emergency management, Wireless Communications IEEE, Vol.15, No.3, (June 2008) page numbers (8-14)

Clausen, T. \& Jacquet, P. (2006). Optimized link state routing protocol (OLSR), RFC 3626, 2006

Cordeiro, W.; Aguiar, E.; Moreira, W.; Abelem, A. \& Stanton, M. (2007). Providing quality of service for mesh networks using link delay measurements, 16th International Conference on Computer Communications and Networks, pp. 991 -996, 2007

De Couto, D.; Aguayo, D.; Bicket, J. \& Morris, R. (2003). A high-throughput path metric for multi-hop wireless routing, 9th Annual International Conference on Mobile Computing and Networking, pp. $134-146,2003$

De Vleeschauwer, B. et al. (2008). End-to-end QoE Optimization Through Overlay Network Deployment. Information Networking, 2008. ICOIN 2008. International Conference on, pp. 1-5, January 2008

Engelke, Ulrich; Zepernick, Hans-Jurgen (2007). Perceptual-based Quality Metrics for Image and Video Services: A Survey. Next Generation Internet Networks, 3rd EuroNGI Conference on, pp.190-197, May 2007.

ETSI (2010). European Technical Committee for Speech, Transmission, Planning, and Quality of Service, accessed in April 2010, of http:/ / portal.etsi.org

Evalvid (2010). Evalvid: A Video Quality Evaluation Tool-set, accessed in March 2010, of http://www.tkn.tu-berlin.de/research/evalvid/

Fall, K. \& Varadhan, K. (2010). The network simulator - ns-2, accessed in August 2010, of http://www.isi.edu/nsnam/ns/

Garcia, M. et al. (2009). A QoE Management System for Ubiquitous IPTV Devices. Mobile Ubiquitous Computing, Systems, Services and Technologies, Third International Conference on, pp.147-152, October 2009, UBICOMM '09

Gavrilovska, L.M.; Atanasovski, V.M. (2005). Ad hoc networking towards 4G: Challenges and QoS solutions. International Conference on Telecommunications in Modern Satellite, Cable and Broadcasting Services, September 2005

Gomes, R. L.; Ferreira Júnior, J.; Moreira Júnior, W. \& Abelém, A. J. (2009). QoE and QoS in Wireless Mesh Networks. IEEE Latin-American Conference on Communications, 2009

Gomes, R.; Moreira Junior, W.; Cerqueira, E. \& Abelem, A. (2009). Using a Fuzzy Link Cost and Dynamic Choice of Metrics to Achieve QoS and QoE in Wireless Mesh Networks. Journal of Network and Computer Applications, pp. 1084-8045, November 2009

Gomes, R.; Moreira, W.; Nascimento, V. \& Abelem, A. (2008). Dynamic metric choice routing for mesh networks, 7th International Information and Telecommunication Technologies Symposium (I2TS), 2008

Greengrass, J.; Evans, J. \& Begen, A.C. (2009). Not All Packets Are Equal, Part I: Streaming Video Coding and SLA Requirements. IEEE Internet Computing, Vol. 13 , No. 1, page numbers $(70-75)$

Held, G. (2005). Wireless Mesh Networks, Auerbach Publications

Hossain, E. \& Leung, K. (2009). Wireless Mesh Networks: Architectures and Protocols, Springer US ITU-R (1995). International Telecommunication Union - Radiocommunication Sector. Technical Report.Series BT: Broadcasting service (television). Recommendation BT.500-7: Methodology for the Subjective Assessment of the Quality of Television Pictures, October 1995 
ITU-T (2010). International Telecommunication Union - Telecommunication Standardization Sector, accessed in April 2010, of http:/ / www.itu.int/ITU-T/

Jain, R. (2004). Quality of experience. IEEE Multimedia, Vol. 11, No.1, 2004, page numbers (95- 96)

Kawadia, V. \& Kumar, P.R. (2005). A cautionary perspective on cross-layer design. Wireless Communications IEEE, Vol.12, No.1, (February 2005) page numbers (3-11)

Kishigami, Jay (2007). The Role of QoE on IPTV Services style, Multimedia, ISM, Ninth IEEE International Symposium on, pp. 11-13, Taichung

Klein, Anja (2007). Incorporating Quality Aspects in Sensor Data Streams, Proceedings of the ACM first Ph.D, Conference on Information and Knowledge Management, pp. 77-84, Lisboa, workshop in CIKM

Kozat, U.C.; Koutsopoulos, I. \& Tassiulas, L. (2004). A framework for cross-layer design of energy-efficient communication with QoS provisioning in multi-hop wireless networks, Twenty-third Annual Joint Conference of the IEEE Computer and Communications Societies, Vol. 2, pp. 1446- 1456, March 2004, INFOCOM 2004.

Lambrecht, C. \& Verscheure, O. (2006). Perceptual Quality Measure Using a SpatioTemporal Model of the Human Visual System. Proceedings of SPIE, Vol. 2668, San Jose, USA.

Monteiro, Janio M.; Nunes, Mario S. (2007). A Subjective Quality Estimation Tool for the Evaluation of Video Communication Systems. Computers and Communications, ISCC 2007, 12th IEEE Symposium on, p. MW - 75 - MW - 80.

Moreira, W.; Aguiar, E.; Abelém, A. \& Stanton, M. (2008). Using multiple metrics with the optimized link state routing protocol for wireless mesh networks. 26th Brazilian Symposium on Computer Networks and Distributed Systems, May 2008

MSU MSU Video Quality Measurement Tool. accessed in August 2010, of http://compression.ru/video/quality measure/index en.html

Pahalawatta, P.; Berry, R.; Pappas, T. \& Katsaggelos, A. (2007). Content-Aware Resource Allocation and Packet Scheduling for Video Transmission over Wireless Networks, Selected Areas in Communications, IEEE Journal on, Vol.25, No.4, (May 2007) page numbers (749-759)

Patrick, Andrew S. et al. (2004). A QoE sensitive architecture for advanced collaborative environments. First International Conference on Quality of Service in Heterogeneous Wired/Wireless Networks, pp. 319-322, October 2004, QSHINE 2004

Pinson, Margaret H.; Wolf, Stephen. (2004). A new standardized method for objectively measuring video quality. IEEE Transactions on Broadcasting, Vol. 50, No. 3, (September 2004) page numbers (312 - 322)

Revés, X.; Nafisi, N.; Ferrús, R. \& Gelonch, A. (2006). User perceived Quality Evaluation in a B3G Network Testbed. IST Mobile Summit, Mykonos, Greece

Romaniak, P.; Mu, M.; Leszczuk, M. \& Mauthe, A. (2008). Framework for the Integrated Video Quality Assessment. Blekinge, Institute of Technology, April 2008, Karlsrona, Sweden

Rowe, Lawrence A. \& Jain, Ramesh (2005). ACM SIGMM Retreat Report on Future Directions in Multimedia Research. ACM Transactions on Multimedia Computing, Communications, and Applications (TOMCCAP), Vol. 1, No. 1, (February 2005) page numbers (3-13) 
Siller, M. \& Woods, J. (2003) Improving Quality of Experience for Multimedia Services by QoS arbitration on QoE Framework. Proceedings of the 13th Packed Video Workshop 2003, April 2003, Nantes, France

Takahashi, A.; Hands, D. \& Barriac, V. (2008). Standardization activities in the ITU for a QoE assessment of IPTV. IEEE Communication Magazine, Vol. 46, No. 2, (February 2008)

Valerdi, J.; Gonzalez, A. \& Garrido, F.J. (2009). Automatic Testing and Measurement of QoE in IPTV Using Image and Video Comparison. Digital Telecommunications, ICDT '09, Fourth International Conference on, pp.75-81, July 2009

V-Factor (2010). V-Factor Quality of Experience Platform, accessed in July 2010, of http://www.pevq.org/

Villalon, J.; Cuenca, P.; Orozco-Barbosa, L.; Seok, Yongho \& Turletti, T. (2007). Cross-Layer Architecture for Adaptive Video Multicast Streaming Over Multirate Wireless LANs, Selected Areas in Communications, IEEE Journal on, Vol.25, No.4, pp.699-711, May 2007

VQEG (2010). Video Quality Experts Group, accessed in April 2010, of http://www.its.bldrdoc.gov/vqeg/

Wang, Zhou; Lu, Ligang; Bovik, Alan C. (2004). Video Quality Assessment based on Structural Distortion Measurement. Signal Processing: Image Communication, Special Issue on Objective Video Quality Metrics, Vol. 19, No. 2, pp. 121-132, February 2004

Wu, D.; Ci, S. \& Wang, H. (2007). Cross-Layer Optimization for Video Summary Transmission over Wireless Networks, Selected Areas in Communications, IEEE Journal on, Vol.25, No.4, pp.841-850, May 2007

Yamada, H. et al. (2007). A QoE based service control scheme for RACF in IP-based FMC networks. E-Commerce Technology and the 4th IEEE International Conference on Enterprise Computing, E-Commerce, and E-Services, CEC/EEE, pp. 611-618

Zapater, Marcio Nieblas; Bressan, Graça (2007). A Proposed Approach for Quality of Experience Assurance of IPTV. First International Conference on the Digital Society, pp. 25-25, Guadeloupe, January 2007, ICDS '07

Zhang, Y.; Luo, J. \& Hu, H. (2006). Wireless Mesh Networking: Architectures, Protocols and Standards, In: Wireless Networks and Mobile Communications Series, Auerbach Publications. 


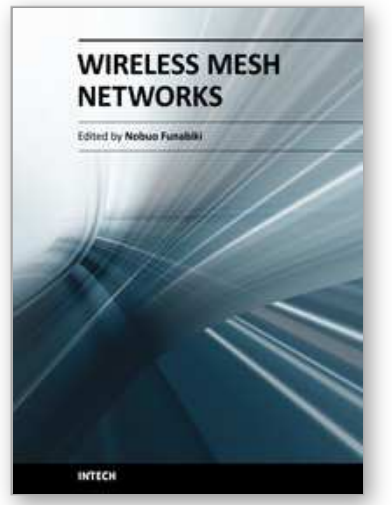

\author{
Wireless Mesh Networks \\ Edited by Nobuo Funabiki
}

ISBN 978-953-307-519-8

Hard cover, 308 pages

Publisher InTech

Published online 14, January, 2011

Published in print edition January, 2011

The rapid advancements of low-cost small-size devices for wireless communications with their international standards and broadband backbone networks using optical fibers accelerate the deployment of wireless networks around the world.â€"The wireless mesh network has emerged as the generalization of the conventional wireless network. However, wireless mesh network has several problems to be solved before being deployed as the fundamental network infrastructure for daily use. The book is edited to specify some problems that come from the disadvantages in wireless mesh network and give their solutions with challenges. The contents of this book consist of two parts: Part I covers the fundamental technical issues in wireless mesh network, and Part II the administrative technical issues in wireless mesh network. This book can be useful as a reference for researchers, engineers, students and educators who have some backgrounds in computer networks, and who have interest in wireless mesh network. It is a collective work of excellent contributions by experts in wireless mesh network.

\title{
How to reference
}

In order to correctly reference this scholarly work, feel free to copy and paste the following:

Elisangela S. Aguiar, Billy A. Pinheiro, João Fabrício S. Figueirêdo, Eduardo Cerqueira, Antônio Jorge. G. Abelém and Rafael Lopes Gomes (2011). Trends and Challenges for Quality of Service and Quality of Experience for Wireless Mesh Networks, Wireless Mesh Networks, Nobuo Funabiki (Ed.), ISBN: 978-953-307519-8, InTech, Available from: http://www.intechopen.com/books/wireless-mesh-networks/trends-andchallenges-for-quality-of-service-and-quality-of-experience-for-wireless-mesh-networks

\section{INTECH}

open science | open minds

\section{InTech Europe}

University Campus STeP Ri

Slavka Krautzeka 83/A

51000 Rijeka, Croatia

Phone: +385 (51) 770447

Fax: +385 (51) 686166

www.intechopen.com

\section{InTech China}

Unit 405, Office Block, Hotel Equatorial Shanghai

No.65, Yan An Road (West), Shanghai, 200040, China 中国上海市延安西路65号上海国际贵都大饭店办公楼 405 单元

Phone: +86-21-62489820

Fax: +86-21-62489821 
(C) 2011 The Author(s). Licensee IntechOpen. This chapter is distributed under the terms of the Creative Commons Attribution-NonCommercialShareAlike-3.0 License, which permits use, distribution and reproduction for non-commercial purposes, provided the original is properly cited and derivative works building on this content are distributed under the same license. 